\title{
Optical visualization and polarized light absorption of the single-wall carbon nanotube to verify intrinsic thermal applications
}

\author{
Xiao Zhang ${ }^{1}$, Li Song ${ }^{2}$, Le Cai ${ }^{1}$, Xuezeng Tian ${ }^{1}$, Qiang Zhang ${ }^{1}$, Xiaoying Qi $^{3}$, Wenbin Zhou ${ }^{1}$, Nan Zhang ${ }^{1}$, \\ Feng Yang ${ }^{1}$, Qingxia Fan ${ }^{1}$, Yanchun Wang ${ }^{1}$, Huaping Liu ${ }^{1}$, Xuedong Bai ${ }^{1}$, Weiya Zhou ${ }^{1}$ and Sishen Xie ${ }^{1}$
}

The predicted extraordinary properties of carbon nanotubes (CNTs) from theoretical calculations have great potential for many applications. However, reliable experimental determination of intrinsic properties at the single-tube level is currently a matter of concern, and many challenges remain because of the unhandled and nanoscale size of individual nanotubes. Here, we demonstrated a prototype to detect the intrinsic thermal conductivity of the single-wall carbon nanotube (SWCNT) and verify the significant non-resonant optical absorption behavior on tiny nanotubes by integrating the nanotube and ice into a new core-shell design. In particular, a reversible optical visualization method based on the individual suspended ultra-long SWCNT was first developed by wrapping a nanotube with ice in the cryogenic air environment. The light-induced thermal effect on the hybrid core-shell structure was used to melt the ice shell, which subsequently acted as a temperature sensor to verify the intrinsic thermal conductivity of the core-like nanotube. More interestingly, we successfully determined for the first time the thermal response phenomenon of the tiny absorption cross section in SWCNT in the vertical-polarization configuration and the significant non-resonant absorption behavior in the parallel-polarization configuration. These investigations will provide a better understanding for the unique optical behaviors of CNT and enable the detection of intrinsic properties of various one-dimensional nanostructures such as nanotubes, nanowires, and nanoribbons.

Light: Science \& Applications (2015) 4, e318; doi:10.1038/Isa.2015.91; published online 14 August 2015

Keywords: carbon nanotube; optical visualization; polarized laser; resonant absorption

\section{INTRODUCTION}

The extraordinary thermal and electrical properties of carbon nanotubes (CNTs) have been extensively investigated in the last decade for applications in various devices. Despite its obvious importance, a reliable experimental determination of the intrinsic properties of SWCNTs, particularly at the single-tube level, remains a challenge because of the tiny sectional area and enormous difficulty in nanotube manipulation, which in fact hampers its wide use. From the theoretical side, an equilibrium molecular dynamic (MD) simulation has suggested that the lattice thermal conductivity of a $(10,10)$ SWCNT reaches the maximum value of approximately $37000 \mathrm{~W}(\mathrm{~K} \cdot \mathrm{m})^{-1}$ at $100 \mathrm{~K}$ and decreases to approximately $6600 \mathrm{~W}(\mathrm{~K} \cdot \mathrm{m})^{-1}$ at $300 \mathrm{~K}^{1}$. However, because of the diversity of measuring methods and sample qualities, the experimentally measured thermal conductivity value $(\kappa)$ significantly varies from $200-25000 \mathrm{~W}(\mathrm{~K} \cdot \mathrm{m})^{-12-5}$. Moreover, in most previously reported measurements, the crystal structure of CNT was not adequately characterized. Consequently, it is predictable that the obtained thermal conductivity values may contain errors that result from the uncertainty in the actual SWCNT cross section, let alone the relationship between structure and thermal property.
Furthermore, experimental results ${ }^{6,7}$ of the polarized optical absorption cross section of SWCNTs in bulk samples provide some direct confirmation of the absorptive process theory ${ }^{8,9}$. Considering that the surrounding environment of CNTs (particularly the substrate and adjacent tubes ${ }^{10,11}$ ) may remarkably affect the properties of CNTs, a reliable experimental determination of the intrinsic properties at the single-tube level is currently a matter of concern. Recent light absorption investigations of individual nanotubes have focused on different resonant and nonresonant modes using spatial modulation spectroscopy ${ }^{12}$, polarization-based homodyne microscope ${ }^{13}$, and photoconductivity spectra ${ }^{14}$. However, to avoid the optical detection difficulty from the small cross section of SWCNTs, the light-induced thermal effect was considered a suitable method to study the thermal and optical properties of CNTs, which is also significant to both the function of optical devices ${ }^{15}$ and SWCNT species control ${ }^{16-18}$. In arrays of nanotubes with mixed structures and chirality, unusual laser-induced "heat trap" phenomena have recently been observed $^{19,20}$. However, intrinsic thermal response study on individual nanotube remains missing because individual CNT's

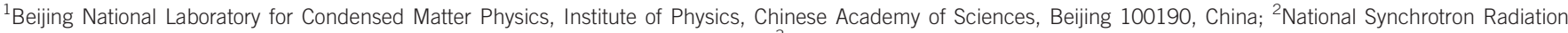
Laboratory, University of Science and Technology of China, Hefei 230029, China and ${ }^{3}$ National Center for Nanoscience and Technology, Beijing 100190, China 
manipulation and visualization are challenging. Recently, a flowguided synthesis method was reported to grow ultra-long nanotubes with perfect crystalline structure (maintaining the chirality without any change for more than hundreds of micrometers ${ }^{21}$ ). Therefore, the ultra-long nanotube is considered an ideal object to characterize intrinsic nanotube properties, particularly the suspended nanotube with unique superiority in avoiding any effects from the substrate $e^{12,22}$.

Moreover, optical microscopic imaging resolution has a theoretical limit of approximately $200 \mathrm{~nm}$ within the visible-light spectrum because of the far-field diffraction limit, which prevents the technique from being used to directly observe nano-objects ${ }^{23}$. If individual ultralong nanotubes can be observed under an optical microscope through a facile and reversible method, nanotube characterization and manipulation will tremendously benefit its fundamental investigations and various specific applications.

In this contribution, the individual and suspended ultra-long SWCNT is successfully synthesized to cross over an as-patterned optical slit. Then, its chirality is characterized using high-resolution transmission electron microscopy (HRTEM), electron diffraction (ED) and Raman spectra. More interestingly, a facile method was proposed to make such suspended SWCNTs visible by depositing ice around them to form a new core-shell structure. We demonstrate that when irradiated by lasers, the ice-enclosed SWCNT can be heated, which melts the ice around it. By analyzing this ice melt strategy and the inferred temperature from the SWCNT's Raman peak shift, we propose a prototype to directly identify the intrinsic thermal conductivity of the individual suspended SWCNT. With its structure and chirality, which are obtained from the analysis of ED and Raman spectra, the relationship between the nanotube structure and its thermal property is further verified. Furthermore, we use laser polarization absorption experiments to reveal, for the first time, the thermal response evidence of a tiny absorption cross section of SWCNTs in the vertical-polarization configuration and its significant non-resonant absorption behavior in the parallel-polarization configuration.

\section{MATERIALS AND METHODS}

\section{Suspended CNT sample preparation}

To study the core-shell structure, first, we prepared the individual suspended SWCNTs using a previously described method ${ }^{24}$. $3 \mathrm{mg} \mathrm{mL}{ }^{-1}$ hemoglobin $(\mathrm{Hb})$ solution was spin-coated on silicon wafers with a $500 \mathrm{~nm}$ thick thermally oxidized layer as the catalyst substrates. Before the growth procedure, the catalyst substrate was annealed in air at $800{ }^{\circ} \mathrm{C}$ for $20 \mathrm{~min}$ and subsequently pretreated at $950{ }^{\circ} \mathrm{C}$ in $\mathrm{H}_{2}$ for 5 min to effectively reduce the catalyst. The nanotube growth was initiated by introducing $25 \mathrm{sccm} \mathrm{H} \mathrm{H}_{2}$ and $10 \mathrm{sccm} \mathrm{CH}_{4}$ at $950{ }^{\circ} \mathrm{C}$. After 20 min growth, the sample was cooled in Ar to room temperature. The entire preparation process was performed at atmospheric pressure.

\section{Raman and melting experiments}

We performed the optical experiment on a HORIBA HR800 microRaman spectroscopy equipped with a Linkam model THMS600 heating and cooling microscope stage to maintain the sample at different constant temperatures in air. The optical setup also includes $50 \times$ objective $($ N.A. $=0.5), 1800$ grating, and lasers with excitation energy of $2.41 \mathrm{eV}(514.5 \mathrm{~nm}), 1.96 \mathrm{eV}(632.8 \mathrm{~nm})$, and $1.58 \mathrm{eV}$ $(785 \mathrm{~nm})$. The largest laser power on the sample is $2.4,4.9$, and $7.5 \mathrm{~mW}$, respectively (measured using an optical power meter).
Substrate preparation and chiral index (n, m) characterization Because of the sensitivity of nanotube's properties to atomic configuration, a reliable method to determine the nanotube structures is indispensable. We determined the atomic structure of each CNT from HRTEM and the ED pattern ${ }^{25}$ using nanofocused electron beams in an FEI Tecnai G2 F20 U-TWIN TEM and JEOL 2010F TEM. To avoid conceivable large drifting and optical experiment uncertainty, which are induced by electron irradiation damage, as illustrated in Figure 1a and $1 \mathrm{~b}$, a homemade substrate was used as a receiving substrate on which the suspended nanotubes were directly grown. Using this new receiving substrate, the electron beam and laser beam can pass through the narrow TEM slits $(5 \mu \mathrm{m} \times 200 \mu \mathrm{m}$ with location marks $)$ and wide optical slit $(100 \mu \mathrm{m} \times 200 \mu \mathrm{m})$ respectively. This design enables the combination of TEM ED and optical spectroscopy techniques to investigate both the chiral index and the optical, thermal properties of the same individual suspended SWCNTs.

\section{RESULTS AND DISCUSSION}

Visualization of SWCNTs via a hybrid ice-SWCNT core-

\section{shell design}

To form the core-shell structure, the substrate with suspended ultralong SWCNTs was loaded into the heating and cooling microscope stage (Figure 1c). When the temperature of the stage decreased, the individual suspended SWCNTs gradually became visible as a wire across the slit under an optical microscope, as shown in Figure 1d and 1e. We identified the Raman spectrum of the shell, which had an $\mathrm{O}-\mathrm{H}$ stretching vibration mode at approximately $3200 \mathrm{~cm}^{-1}$. Thus, we ascertained that, as long as the temperature was sufficiently low, the supercooling water vapor in the surrounding would rapidly condense onto the nanotubes to form a unique SWCNT-ice core-shell structure of a few micrometers. The hybrid structure enables the individual SWCNT to be visualized as a wire under the optical microscope, although the CNT has a notably tiny diameter of approximately $2 \mathrm{~nm}$.

Similar efforts have been reported to make CNT visible under an ordinary optical microscope to easily manipulate CNTs, such as electroplating $\mathrm{Au}$ nanoparticles ${ }^{26}$ and depositing $\mathrm{TiO}_{2}$ particles ${ }^{27}$. Compared with the previous methods, in our strategy, the visualization process is reversible because the nanotube will recover to its naked form without any change in structure when it returns to room temperature (proven by comparing the TEM feature and ED pattern before and after the melting process). Very recently, Wang et al. developed a technique to observe nanostructures using an optical microscope with water vapor condensation ${ }^{28}$. Here, we want to point out that in our method, the nanotube attached to the substrate can also be reversibly visualized probably because ice nucleation was facilitated by the nanotubes in comparison to the surrounding flat substrate. However, a remarkable difference between our method and that of Wang et al. is how to construct a supercooling water vapor environment. Thanks to this distinction, our method can maintain the visibility of the CNTs as long as the temperature is sufficiently low. More importantly, this distinction endows the ice-CNT structure some further intriguing functionalities as follows.

\section{Polarization laser-induced melt experiment}

The most intriguing phenomenon is that when exposed to a parallelpolarized laser with intensity of several $\mathrm{mW} \mu \mathrm{m}^{-2}$, the wire began to gradually vanish from the irradiated position to two ends and formed a melt section, as shown in Figure $2 \mathrm{a}$ and $2 \mathrm{~b}$. The melt section was always much larger than the laser spot, which is calculated using the diffraction limit $\left(1.2 \frac{\lambda}{\text { N.A. }}\right.$, which is calculated to be $1.2,1.5$, and 

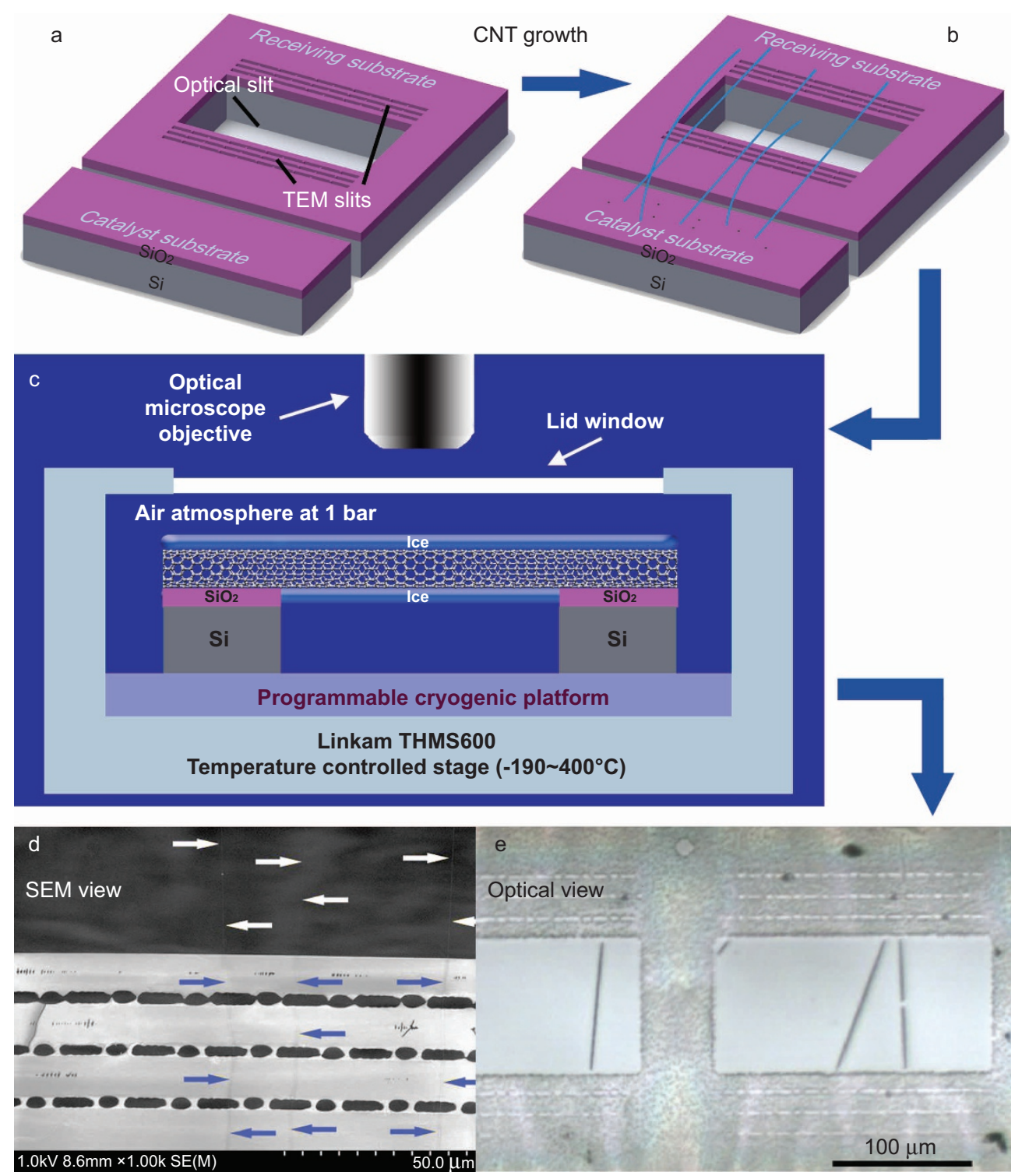

Figure 1. (a-b) A schematic illustration of the controllable preparation of an individual suspended SWCNT sample using a flow-guided chemical vapor deposition method. (c) The illustration shows the formation of the new core-shell structure by depositing ice around the SWCNTs, when the temperature-controlled stage maintained a cryogenic air environment. (d) An SEM image of suspended nanotubes that cross over the optical slit (indicated by white arrows) and three TEM slits (indicated by blue arrows). (e) Suspended nanotubes coated with ice became visible under an optical microscope as wires across the optical slits.

$1.9 \mu \mathrm{m}$ for 514,633 , and $785 \mathrm{~nm}$ lasers, respectively). The margin between the ice-enclosing part and the naked part, which we call the melt edge, would reach a steady site after several minutes of exposure.

To determine whether the laser-inducing melt phenomenon originates from the heating of the CNT instead of the ice itself, we rotated the sample by $90^{\circ}$ to make the axis of the SWCNT perpendicular with the incident laser polarization. Because of the deep sub-wavelength and highly anisotropic dimensions of SWCNTs, the interaction between SWCNTs and light strongly depends on the orientation of the electric field with respect to the SWCNT axis. In contrast, the environment and ice around the SWCNT feels no difference with the laser polarization. As shown in Figure 2c, almost no melt can be observed after the middle of the suspended core-shell structure was irradiated with laser for $15 \mathrm{~min}$. However, after adding a $\lambda / 2$ retardation plate to turn the laser polarization parallel to the SWCNT axis and irradiating the wire for $5 \mathrm{~min}$, an obvious melt section was clearly observed around the laser spot as shown in Figure 2d, despite the decrease in laser intensity with the $\lambda /$ 2 retardation plate in the light path. According to the above observations, the melt phenomenon undoubtedly originates from heating the SWCNT despite its nanoscale size.

We have looked into our samples using an optical microscope and estimated the thickness range of the ice shell, which is approximately 1-2 $\mu \mathrm{m}$. In other words, the ice shell is notably thick (almost three orders larger than that of the SWCNT diameter). Thus, we suggest that the ice shell should have a notably lower degree of polarization effect than SWCNT.

\section{Theoretical basis}

As previously mentioned, the enclosed SWCNT in ice was heated by the laser. The ice began to gradually melt from the irradiated position to two sides until a dynamic equilibrium was established. Then, the power of the laser input to SWCNTs was offset by the power loss transferred from 


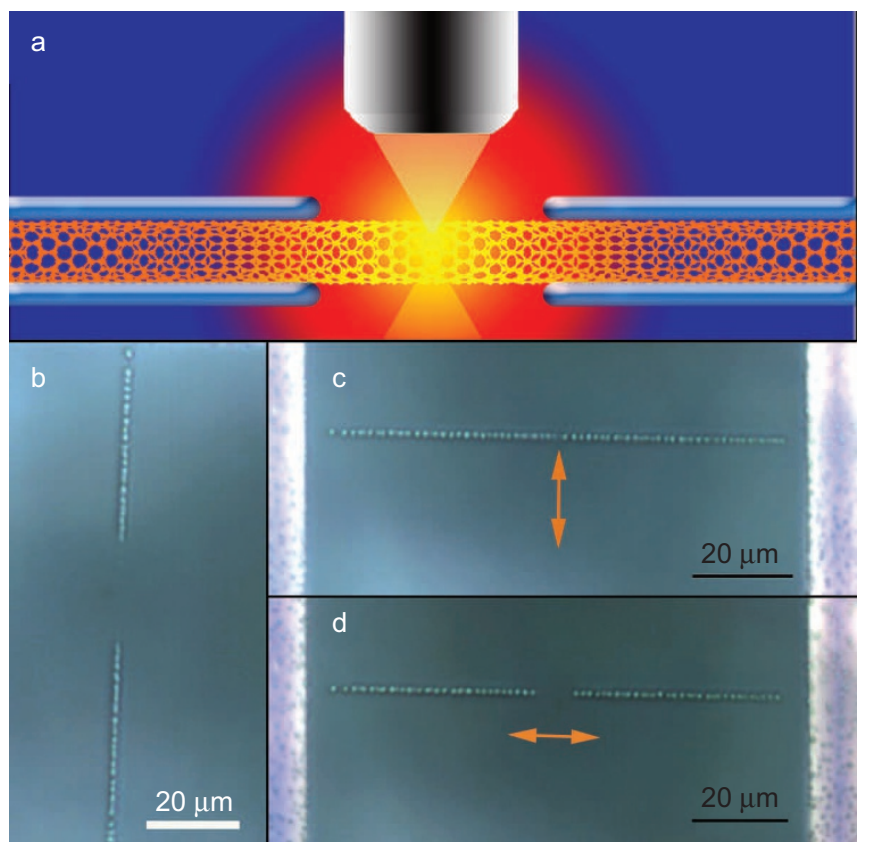

Figure 2. (a) A schematic illustration of the heat-induced melt of an SWCNT-ice core-shell wire from laser irradiation. (b) A laser-induced melt section was experimentally formed around the irradiated spot, when the SWCNT-ice core-shell wire was exposed to a parallel-polarized $785 \mathrm{~nm}$ laser with a power of $7.5 \mathrm{~mW}$ for $12 \mathrm{~min}$. (c) In the vertical-polarization configuration, no melt section can be observed even after laser irradiating for $15 \mathrm{~min}$. (d) In the parallel-polarization configuration, an obvious melt section can be directly observed near the laser spot with a much weaker laser intensity than that used for the vertical-polarization configuration.

SWCNTs to the surrounding gas molecules or ice out of the melt region. Meanwhile, because the nanotube temperature decreased to nearly the surrounding temperature within a short distance from the laser spot, we could neglect the heat lost through the ends of the SWCNT.

Therefore, the spatial temperature profile can be analyzed using two sectionalized stationary one-dimensional (1D) heat diffusion equations, which describe the naked and enclosed sections, respectively.

The naked part can be expressed in Equation (1):

$$
\kappa \frac{\mathrm{d}^{2} T}{\mathrm{~d} x^{2}}-\frac{g P}{A}\left(T-T_{0}\right)=0
$$

where $T, T_{0}, g, P, A$, and $\kappa$ represent the local temperature of SWCNT, ambient temperature of the surrounding gas environment, heat transfer coefficient between SWCNTs and the gas, SWCNT's perimeter, cross-sectional area, and thermal conductivity, respectively.

We assumed that the temperature of SWCNT at the margin of the laser spot was $T_{\mathrm{H}}$. Here, ice was actually considered a temperature sensor, which had a fixed temperature $T_{M}$ at melt edge $x_{M}$. Using these boundary conditions, we obtained the solution of Equation (1):

$$
\begin{array}{r}
T(x)=a e^{m x}+b e^{-m x}+T_{0} \text { and } m=\sqrt{\frac{g P}{\kappa A}}, a=\frac{\Delta T_{\mathrm{M}}-\Delta T_{\mathrm{H}} e^{-m x_{\mathrm{M}}}}{e^{m x_{\mathrm{M}}}-e^{-m x_{\mathrm{M}}}}, \\
b=\frac{\Delta T_{\mathrm{H}} e^{m x_{\mathrm{M}}}-\Delta T_{\mathrm{M}}}{e^{m x_{\mathrm{M}}}-e^{-m x_{\mathrm{x}}}}, \Delta T_{\mathrm{H}}=T_{\mathrm{H}}-T_{0}, \Delta T_{\mathrm{M}}=T_{\mathrm{M}}-T_{0}
\end{array}
$$

The enclosed part can be expressed in Equation (3):

$$
\kappa \frac{\mathrm{d}^{2} T}{\mathrm{~d} x^{2}}-\frac{g^{\prime} P}{A}\left(T-T_{0}\right)=0
$$

where $g^{\prime}$ represents the heat transfer coefficient between ice and SWCNT. Similar to the aforementioned statement, the SWCNT temperature of the melt edge is $T_{\mathrm{M}}$. Because the nanotube temperature declines to nearly the surrounding temperature within a short distance (usually within several micrometers), we obtain $T(\infty)=T_{0}$. Using the above boundary conditions, the solution of Equation (3) is as follows:

$$
\begin{aligned}
& T(x)=\Delta T_{\mathrm{M}} e^{-m^{\prime} x}+T_{0} \\
& \text { and } m^{\prime}=\sqrt{\frac{g^{\prime} P}{\kappa A}}
\end{aligned}
$$

Because the temperature profile along the SWCNT is differentiable, the first derivatives of above two solutions must be equal at the melt edge. Thus, we obtain:

$$
-m^{\prime} \Delta T_{\mathrm{M}} e^{-m^{\prime} \times 0}=m a e^{m x_{\mathrm{M}}}-m b e^{-m x_{\mathrm{M}}}
$$

The solution of Equation (5) is

$\left(\sqrt{\frac{g^{\prime}}{g}} \frac{\Delta T_{\mathrm{M}}}{\Delta T_{\mathrm{H}}}+\frac{\Delta T_{\mathrm{M}}}{\Delta T_{\mathrm{H}}}\right) \mathrm{Y}^{2}-2 \mathrm{Y}+\left(-\sqrt{\frac{g^{\prime}}{g}} \frac{\Delta T_{\mathrm{M}}}{\Delta T_{\mathrm{H}}}+\frac{\Delta T_{\mathrm{M}}}{\Delta T_{\mathrm{H}}}\right)=0$

and $\mathrm{Y}=e^{m x_{\mathrm{M}}}$

Based on the above analysis, we obtain the relationship between $\Delta T_{\mathrm{H}}, x_{\mathrm{M}}$, and $\kappa$, which can be used to measure its thermal conductivity and analyze the light absorption ability of SWCNTs.

\section{Thermal conductivity measurement}

According to the Equation (6), the thermal conductivity of SWCNT can be calculated based on $g^{\prime}, g, \Delta T_{\mathrm{M}}, \Delta T_{\mathrm{H}}, x_{\mathrm{M}}$ and the geometry of tube, $P$ and $A$.

As mentioned above, $P$ is the outside perimeter of the CNT, which transfers heat to the surroundings, and $A$ is the cross-sectional area of the CNT, which transfers heat along the axis of the CNT. $A$ is calculated in previous reports ${ }^{29} . A \equiv \sum_{j}^{n} \pi d_{j} \delta=n \pi \delta\left[d_{j}+(n-1) \delta\right]$, where $n$ is the number of shells in the CNT, $d_{j}$ is the diameter of the $j$-th nanotube shell, and $\delta=0.335 \mathrm{~nm}$ is the interplanar spacing of graphite.

For SWCNT, the upper equation reduces to $A=\pi d \delta$ and $P=\pi d$. The $A$ of SWCNT also accounts for the cross-sectional area of each shell in double-wall carbon nanotube (DWCNT) and multi-wall carbon nanotube (MWCNT) samples.

It is worth noting that although the laser power is the heat source of the melting, we avoid measuring or calculating the transferred laser power to the CNTs, which was proven to be difficult in previous reports. Instead, $\Delta T_{\mathrm{H}}$ was measured and calculated based on the heat-induced Raman $\mathrm{G}$ band red-shift ${ }^{24}$, which is frequently used to identify the temperature of carbon nano-materials. In addition, $\Delta T_{\mathrm{H}}$ has a positive correlation with the transferred power.

The fixed melt temperature $T_{\mathrm{M}}$ can be identified by directly notably slowly increasing the temperature of the programmed stage until the ice-CNTs structure becomes invisible again. As previously mentioned, $T_{\mathrm{M}}=-87{ }^{\circ} \mathrm{C}$, so $\Delta T_{\mathrm{M}}=13 \mathrm{~K}$ when $T_{0}=-100{ }^{\circ} \mathrm{C}$. Because of the symmetrical configuration, $x_{\mathrm{M}}$ can be calculated by subtracting the calculated laser spot radius from half of the melt length, which is measured under an optical microscope.

We characterized the atomic structure of each CNT from the HRTEM (Figure 3a) and ED pattern ${ }^{25}$ (Figure $3 \mathrm{~b}$ and $3 \mathrm{c}$ ). The HRTEM observations indicated that the diameter of the as-grown SWCNTs is $1-3 \mathrm{~nm}$. We characterized the nanotube structure at both 

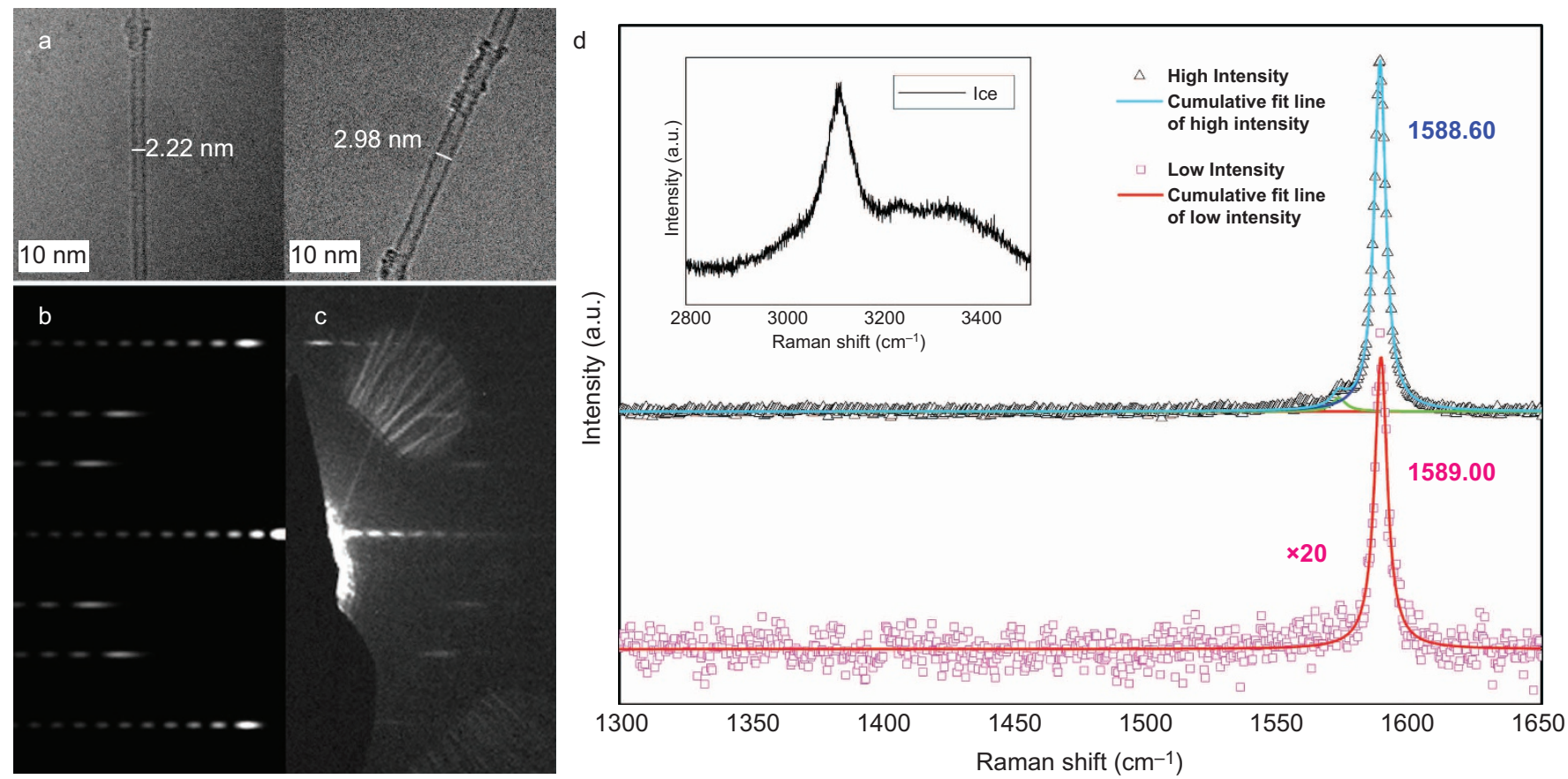

Figure 3. (a) High-resolution transmission electron microscopy images of two individual SWCNT with diameters of 2.22 and $2.98 \mathrm{~nm}$. The wall numbers and diameter of all samples were determined from the ED pattern and verified using the HRTEM image. (b-c) Standard and experimental ED pattern of a $(21,4)$ nanotube. (d) D and $G$ bands of the Raman spectra for the $(21,4)$ nanotube, which was excited by $514 \mathrm{~nm}$ laser with high and low intensity. The negligible D band at approximately $1320 \mathrm{~cm}^{-1}$ illustrates the perfect crystalline of the as-grown suspended ultra-long SWCNT. All peaks were fitted by a Lorentzian curve to distinguish the G band frequency and heat-induced red-shift (inset). Raman spectra of the ice O-H stretching vibration mode.

higher and lower narrow slits to guarantee that nanotubes preserve its chirality without any change in ED patterns. We further verified its atomic structure using its Raman resonant condition based on the $\mathrm{G}$ band intensity. In other words, if $\mathrm{E}_{\text {laser }}$ lies in the $\mathrm{G}$ band resonance window of $\mathrm{E}_{\mathrm{ii}}$, the $\mathrm{G}$ band intensity will be enormously enhanced, not vice-versa. In addition, there are also DWCNT and bundles of SWCNTs, of which the ED patterns come from all inner nanotubes. Therefore, it is difficult to accurately determine the SWCNTs in DWCNT and bundles.

In Table 1, we listed all CNT structures or chirality, and the corresponding reported $\mathrm{E}_{\mathrm{ii}}{ }^{30}$ To study the light-induced melt phenomenon, we also repeated the above melt experiment using 514, 633, and $785 \mathrm{~nm}$ lasers and concluded the melt condition in Table 1.

As the temperature coefficient of red-shift, $\alpha_{\omega}=\left(\frac{\mathrm{d} \omega}{\mathrm{d} T}\right)_{100 \mathrm{~K}} / \omega_{100 \mathrm{~K}}$, does not change at specific temperature. $\Delta T_{\mathrm{H}}$ can be calculated from the $\mathrm{G}$ band red-shift frequency. Using this method, we can prevent large-heat capacity objects from touching the SWCNTs for the local temperature measurement. However, it is noteworthy that the G band would be red-shifted when the nanotubes were wrapped in ice, which we believe to originate from the stretch of the nanotube by the ice gravity. To avoid the effect of ice, we first irradiated the wire with the highest laser intensity for more than $10 \mathrm{~min}$ to form a steady melt. Then, we acquired the red-shifted Raman G band signal at the identical laser intensity. After the SWCNTs cooled, the normal G band was acquired again with a laser intensity smaller than $0.1 \mathrm{~mW} \mu \mathrm{m}^{-2}$ to avoid the thermal effects (Figure $3 \mathrm{~d}$ ). The calculated $\Delta T_{\mathrm{H}}$ of each CNT is shown in Table 1 (the values in the parentheses). As shown in Table 1, the temperature range of our experiments is $170-350 \mathrm{~K}$, where the value of $\kappa$ changes notably slowly as previously reported ${ }^{31}$.
Therefore, it is suitable to take $\kappa$ as a constant for each individual CNT in our calculation.

The thermal boundary conductance (TBC) between the surface of the CNTs and various molecules has been theoretically investigated in several reports. Because the diameter of the SWCNT is in the nanometer scale, the TBC calculation should be based on the kinetic theory of molecules instead of free convection or other continuum approximations. Hu et al. simulated the TBC between a CNT and air as $0.1 \mathrm{~mW}\left(\mathrm{~m}^{2} \cdot \mathrm{K}\right)^{-1}$ at $1 \mathrm{~atm}^{32}$, which is consistent with additional MD simulations using a simple gas kinetic model. MD simulations have predicted the TBC of a SWCNT surrounded by liquid molecules as $1.6 \mathrm{~mW}\left(\mathrm{~m}^{2} \cdot \mathrm{K}\right)^{-133}$. Here, we use $\mathrm{g} \approx 0.1 \mathrm{~mW}\left(\mathrm{~m}^{2} \cdot \mathrm{K}\right)^{-1}$ and $\mathrm{g}^{\prime} \approx 1.6 \mathrm{~mW}\left(\mathrm{~m}^{2} \cdot \mathrm{K}\right)^{-1}$.

Based on the above discussion, we proposed a prototype to calculate the thermal conductivity of each CNT and summarized the results in Figure 4. As shown, the thermal conductivity of SWCNTs is approximately $4000 \mathrm{~W}(\mathrm{~K} \cdot \mathrm{m})^{-1}$ at approximately $-50{ }^{\circ} \mathrm{C}$ in our original steps. We attribute this appealing value to the following factors: (i) ultralong SWCNTs that are grown using the flowing mechanism have a nearly ideal crystalline structure, which eliminates unnecessary scattering by defects; (ii) the suspended naked SWCNTs have an increase in thermal conductivity of over $30 \%$ compared with the supported SWCNTs because of the elimination of scattering at the substrateSWCNT interface ${ }^{34}$; (iii) our method avoids difficult measurements of the input power and thermal boundary resistance (TBR); and (iv) possible overestimation of $\mathrm{g}^{\prime}$ in our ice-enclosing configuration. Moreover, $\kappa$ is reported to decrease with increasing temperature because of anharmonic interactions that produce phonon-phonon Umklapp scattering ${ }^{31}$. 
Table 1. Structure property and melt condition (temperature rise) under 514, 633, and $785 \mathrm{~nm}$ laser irradiation in the parallelpolarization configuration

\begin{tabular}{|c|c|c|c|c|c|c|}
\hline No. & Chirality & $d / \mathrm{nm}$ & $\mathrm{E}_{\mathrm{i} i} / \mathrm{eV}^{\mathrm{a}}$ & $\begin{array}{c}\text { Melt length }\left(\Delta T_{H}\right) \text { under } \\
514 \text { laser } / \mu \mathrm{m}(/ \mathrm{K})^{\mathrm{b}}\end{array}$ & $\begin{array}{l}\text { Melt length }\left(\Delta T_{H}\right) \text { under } \\
633 \text { laser/ } \mu \mathrm{m}(/ \mathrm{K})^{\mathrm{b}}\end{array}$ & $\begin{array}{c}\text { Melt length }\left(\Delta T_{H}\right) \text { under } \\
785 \text { laser/ } \mu \mathrm{m}(/ \mathrm{K})^{\mathrm{b}}\end{array}$ \\
\hline a & $(21,4)$ & 1.82 & $0.58,0.99,2.09,2.29,3.59$ & $4(32)$ & 5 (N.A.) & 7 (N.A.) \\
\hline$b 1^{c}$ & $(18,15)$ & 2.24 & $1.23,1.25,2.36,2.41,3.36$ & $8(85)$ & 10 (N.A.) & 18 (N.A.) \\
\hline$b 2^{c}$ & $(18,15)$ & 2.24 & $1.23,1.25,2.36,2.41,3.36$ & $6(46)$ & 4 (N.A.) & 14 (N.A.) \\
\hline $\mathrm{cl}^{\mathrm{c}}$ & $(18,15)$ & 2.24 & $1.23,1.25,2.36,2.41,3.36$ & $13(170)$ & 7 (N.A.) & 18 (N.A.) \\
\hline$c 2^{c}$ & $(18,15)$ & 2.24 & $1.23,1.25,2.36,2.41,3.36$ & $12(165)$ & 4 (N.A.) & 14 (N.A.) \\
\hline d & $(29,13)$ & 2.91 & $0.36,0.68,1.29,1.64,2.13,2.56$ & $6(45)$ & $10(24)$ & 20 (N.A.) \\
\hline e & $(24,20)$ & 2.98 & $0.36,0.66,1.27,1.57,2.12,2.43$ & $7(29)$ & 7 (N.A.) & 19 (310) \\
\hline$f^{e}$ & $(37,15)$ & 3.68 & & $7(32)$ & $10(3)$ & $11(85)$ \\
\hline Bundle-a & Bundle of 2 SWCNT & $\sim 4.0^{f}$ & & $5(28)$ & 3 (N.A.) & 23 (N.A.) \\
\hline Bundle-b & Bundle of 2 SWCNT & $\sim 4.4^{f}$ & & $12(63)$ & $13(66)$ & $13(157)^{d}$ \\
\hline DWCNT-a & DWCNT & $\sim 1.5^{f}$ & & $10(47)$ & 7 (N.A.) & 13 (N.A.) \\
\hline
\end{tabular}

${ }^{a} E_{i i}$ (lists from $E_{11}$ to $E_{55}$ or $E_{66}$ ) of each SWCNT is applied to room temperature. ${ }^{30}$

${ }^{\mathrm{b}}$ The numbers outside the parentheses are the melt lengths, which were measured under an optical microscope. The numbers in parentheses are the relative temperature increase of the CNT at the margin of the laser spot. $\Delta T_{H}$ values that are expressed as N.A. cannot be acquired because the G band does not resonate with the photon energy. All melt length and temperature increase data are the average results of repeated melt and measurements.

${ }^{c} \mathrm{c} 1$ and $\mathrm{c} 2$ come from one identical ultra-long SWCNT but different optical slits. The same is true for $\mathrm{d} 1 \mathrm{and} \mathrm{d} 2$.

${ }^{\mathrm{d}}$ Operated at $-120^{\circ} \mathrm{C}$.

${ }^{\mathrm{e}}$ This SWCNT's diameter is so large that we have not found its $\mathrm{E}_{\mathrm{ii}}$ value in previous reports.

f Outer diameter of the DWCNT or bundles under HRTEM.

Another controversial thermal conductivity factor is its diameter dependence. In Figure 4, we can see that $\kappa$ tends to increase with the increase in SWCNT diameter in our prepared samples, which coincides with new theory reports ${ }^{34,35}$ (the diameter-dependent thermal conductivity difference is mainly because of the curvature-induced phonon relaxation time shortening ${ }^{34}$, or it is attributed to the presence of additional low- and moderate-frequency radial breathing modes in larger-diameter nanotubes ${ }^{35}$ ).

It is noteworthy that for DWCNT and SWCNT bundles, whose ED patterns come from all inner nanotubes, an accurate determination of all SWCNT indices is difficult. Without an accurate estimation of the cross-sectional area and surface area of these CNTs, the thermal con-

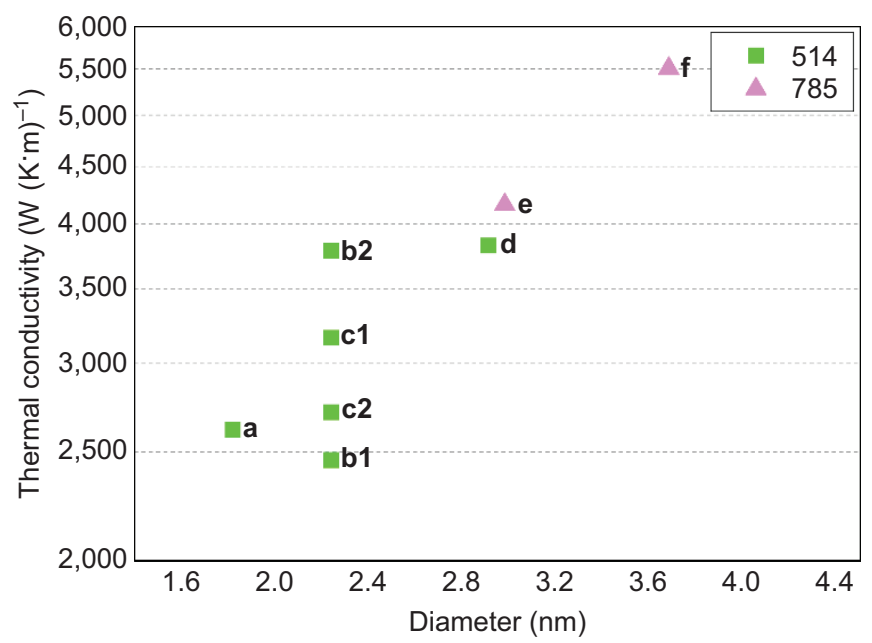

Figure 4. Calculated thermal conductivity of each CNT vs. diameter. The different $\kappa$ of $\mathrm{c} 1, \mathrm{c} 2, \mathrm{~b} 1$, and $\mathrm{b} 2$ under different lasers are within the measurement uncertainties. ductivity cannot be derived. Further elaborate experiments are in progress.

Because the diameter of the prepared SWCNT is confined between 1.8 and $4.0 \mathrm{~nm}$, and the number of samples is limited, a more comprehensive conclusion could only be made based on extra elaborate experiments, particularly considering the following extra points:

Unlike previously reported methods, the ice melt strategy with inferred temperature from the Raman shift can reflect the inherent thermal response, which suppresses the measurement disturbance. In addition, the tough measurement or calculation of the transferred power of laser to CNT is avoided. Therefore, we suggest that this prototype can be facile to verify the intrinsic thermal property of nanotubes and lead to a more convincing result.

Furthermore, the melt distances may fluctuate during repeated melt experiments. This deviation of the melt distance originates from (i) the thickness difference of ice and (ii) the focus condition of the Gaussian profile laser beam on the core-shell structure. Although this deviation obstructs the assessment of the CNT absorption ability to some extent, it will exert a negligible effect on the calculation of the thermal conductivity because the temperature of the SWCNT changes with the melt distance, i.e., the self-adjusting property. In fact, a bad focus condition can hardly be observed because of the visualization of iceCNTs structure.

We must admit that the proposed prototype to measure $\kappa$ is an initial attempt, and there are some aspects to be improved. First, we may use other volatile materials to replace ice to form a more controllable and thinner shell structure around 1D materials. Even the cryogenic environment can also be avoided. By adopting other volatile materials, we can also enlarge the melt length by performing the measurement in vacuum ${ }^{36}$; thus, the length measurement error can be reduced. Furthermore, because the deviation of $\kappa$ mainly comes from the error of $\Delta T_{\mathrm{H}}$, the results will fluctuate less when the CNT reaches a higher $\Delta T_{\mathrm{H}}(200-400 \mathrm{~K}$ is preferred). To attain this goal, a laser with higher power is essential. It is predicted that this method can also be used with other $1 \mathrm{D}$ nanomaterials. 


\section{Resonant and non-resonant absorption}

In recent years, significant studies have been conducted using CNTs for broadband absorber coatings ${ }^{37}$. Previous theories and experiments demonstrated that optical transitions in SWCNTs arise from excitons with strong binding energy instead of free electrons and holes. Each of these optical resonances can be referred to as $S_{i j}$ or $M_{i j}$ depending on the nature of the nanotube ( $\mathrm{S}$ for semiconducting and $\mathrm{M}$ for metallic) and the corresponding valence (i) and conduction (j) subbands. When the incident light polarizes in parallel to the nanotube axis, transitions occur between the sub-bands with $i=j$, and crosspolarized light addresses the transitions between sub-bands with $|\mathrm{i}-\mathrm{j}|=1$. After the optical transition, the energy of the exciton is efficiently transferred to the crystal lattice through strong electronphonon interactions, which increases the temperature of the SWCNT $^{15}$. It is noteworthy that although the listed $\mathrm{E}_{\mathrm{ii}}$ value in Table 1 is applied at room temperature, $\mathrm{E}_{\mathrm{ii}}$ only increases by approximately $0.05 \mathrm{eV}$ from room temperature to $-100{ }^{\circ} \mathrm{C}^{38}$, which has little effect on the resonant condition.

As observed from the vertical-polarization melt experiment, there was nearly no absorption when the laser polarization was perpendicular to the CNT axis. As recently reported ${ }^{12,13}$, the perpendicular-polarization absolute absorption cross-sectional spectra show small and finite perpendicular absorption (approximately $1 / 4^{13}$ or $<1 / 10^{12}$ of parallel polarization one), and they are largely featureless ${ }^{13}$. However, the parallel-polarization spectra show prominent and different exciton transition peaks, which provide rich information on chirality-dependent nanotube photophysics. Our results verified this conclusion in a thermal response method for the first time. In particular, the difficult signal collection because of the small cross section of the SWCNT can be avoided.

For the parallel-polarization configuration, the resonant SWCNT tended to melt longer with higher $\Delta T_{\mathrm{H}}$. We attributed this phenomenon to the larger absorption cross section when the photon energy matched the gap between Van Hove singularities of the Density of State, which is one of the reasons why identical nanotubes have different melt lengths under 514, 633, and $785 \mathrm{~nm}$ laser. However, the ice also melted when the non-resonant absorption occurred, which resulted from the continuum absorption (including band-to-band transitions, phonon sidebands and higher-order exciton transition). This non-resonant absorption is similar to graphene's universal absorption of approximately $2.3 \%$ in the visible and near-infrared range $^{39}$. Furthermore, we note that this continuum absorption constitutes a significant portion of the total nanotube absorption oscillator strength, which was recently appreciated ${ }^{13}$. However, as shown in Table 1, the effect of the nanotube diameter on the heating length is not obvious. Moreover, we did not notice any obvious difference in either resonant or non-resonant absorption between metallic and semiconducting SWCNTs.

The use of laser light to manipulate SWCNTs is an attractive challenge ${ }^{40}$. Because the photons directly access the electronic energy structures of SWCNTs in a resonant manner, it may be possible to selectively manipulate SWCNTs based on their chirality. According to our results, to reduce the portion of metallic SWCNTs in a mixed sample, we should consider two factors: (i) a proper laser photon energy that matches the resonant absorption metallic SWCNTs instead of semiconducting SWCNTs and (ii) an appropriate gas and temperature that are conducive to make metallic SWCNTs susceptible to oxidation ${ }^{18}$.

\section{CONCLUSIONS}

In summary, the facile and reversible optical visualization of individual suspended ultra-long SWCNTs was realized by wrapping them with ice in a cryogenic air environment. When this core-shell structure is irradiated with a high-power laser, the heated SWCNTs melt the ice around it. By modeling this process and regarding ice as a temperature sensor, a link among the melt length, rising temperature and thermal conductivity is established. With the calculated temperature increase based on the Raman red-shift, our prototype indicates that the intrinsic thermal conductivity of these perfect crystalline SWCNTs is approximately $4000 \mathrm{~W}(\mathrm{~K} \cdot \mathrm{m})^{-1}$. Based on the identified CNT's structure and chirality with HDTEM and ED, we find that $\kappa$ tends to grow with the increase in the SWCNT diameter. Moreover, we also provide a perspective to further improve our prototype. Finally, the thermal response evidence of tiny absorption cross section of SWCNTs in the vertical-polarization configuration and the significant non-resonant absorption in the parallel-polarization configuration were discussed. The prototype shown here can also be used with other 1D nanomaterials to provide new insight into the unique fundamental comprehension of lightmatter interaction in quasi 1D nanomaterials and enable the absolute determination of their thermal properties.

\section{ACKNOWLEDGEMENTS}

This work was supported by the National Basic Research Program of China (Grant Nos. 2012CB932302, 2014CB848900), National Natural Science Foundation of China (U1232131, 90921012, 51172271, 51372269 and 11375198), "Strategic Priority Research Program" of the Chinese Academy of Sciences (XDA09040202), Beijing Municipal Education Commission (Grant No. YB20108000101), and Fundamental Research Funds for the Central Universities (WK2310000035). Li Song thanks the recruitment program of global experts and the CAS Hundred Talent Program of China. The authors thank M. Habib from University of Science and Technology of China for his useful discussion.

1 Berber S, Kwon YK, Tománek D. Unusually high thermal conductivity of carbon nanotubes. Phys Rev Lett 2000; 84: 4613-4616.

2 Kim P, Shi L, Majumdar A, McEuen PL. Thermal transport measurements of individual multiwalled nanotubes. Phys Rev Lett 2001; 87: 215502.

3 Fujii M, Zhang X, Xie HQ, Ago H, Takahashi K et al. Measuring the thermal conductivity of a single carbon nanotube. Phys Rev Lett 2005; 95: 065502.

4 Brown E, Hao L, Gallop JC, Macfarlane JC. Ballistic thermal and electrical conductance measurements on individual multiwall carbon nanotubes. Appl Phys Lett 2005; 87: 023107.

5 Yang DJ, Zhang Q, Chen G, Yoon SF, Ahn J et al. Thermal conductivity of multiwalled carbon nanotubes. Phys Rev B 2002; 66: 165440.

6 Islam MF, Milkie DE, Kane CL, Yodh AG, Kikkawa JM. Direct measurement of the polarized optical absorption cross section of single-wall carbon nanotubes. Phys Rev Lett 2004; 93: 037404.

7 Motavas S, Ivanov A, Nojeh A. The effect of light polarization on the interband transition spectra of zigzag carbon nanotubes and its diameter dependence. Physica E 2014; 56: 79-84.

8 Machón M, Reich S, Thomsen C, Sánchez-Portal D, Ordejón P. Ab initio calculations of the optical properties of 4-angstrom-diameter single-walled nanotubes. Phys $\operatorname{Rev} B$ 2002; 66: 155410.

9 Moghaddam MV, Yaghoobi P, Nojeh A. Polarization-dependent light-induced thermionic electron emission from carbon nanotube arrays using a wide range of wavelengths. App/ Phys Lett 2012; 101: 4772504

10 Raravikar NR, Keblinski P, Rao AM, Dresselhaus MS, Schadler LS et al. Temperature dependence of radial breathing mode Raman frequency of single-walled carbon nanotubes. Phys Rev B 2002; 66: 235424.

11 Fantini C, Jorio A, Souza M, Strano MS, Dresselhaus MS et al. Optical transition energies for carbon nanotubes from resonant Raman spectroscopy: environment and temperature effects. Phys Rev Lett 2004; 93: 147406. 
12 Blancon JC, Paillet M, Tran HN, Than XT, Guebrou SA et al. Direct measurement of the absolute absorption spectrum of individual semiconducting single-wall carbon nanotubes. Nat Commun 2013; 4: 2542.

13 Liu K, Hong X, Choi S, Jin C, Capaz RB et al. Systematic determination of absolute absorption cross-section of individual carbon nanotubes. Proc Natl Acad Sci 2014; 111: 7564-7569.

14 DeBorde T, Aspitarte L, Sharf T, Kevek JW, Minot ED. Determining the chiral index of semiconducting carbon nanotubes using photoconductivity resonances. J Phys Chem C 2014; 118: 9946-9950.

15 Itkis ME, Borondics F, Yu AP, Haddon RC. Bolometric infrared photoresponse of suspended single-walled carbon nanotube films. Science 2006; 312: 413-416.

16 Kocabas C, Meitl MA, Gaur A, Shim M, Rogers JA. Aligned arrays of single-walled carbon nanotubes generated from random networks by orientationally selective laser ablation. Nano Lett 2004; 4: 2421-2426.

17 Maehashi K, Ohno Y, Inoue K, Matsumoto K. Chirality selection of single-walled carbon nanotubes by laser resonance chirality selection method. Appl Phys Lett 2004; 85: 858-860.

18 Huang HJ, Maruyama R, Noda K, Kajiura H, Kadono K. Preferential destruction of metallic single-walled carbon nanotubes by laser irradiation. J Phys Chem B 2006; 110(14): 7316-7320

19 Yaghoobi P, Moghaddam MV, Nojeh A. "Heat trap": light-induced localized heating and thermionic electron emission from carbon nanotube arrays. Solid State Commun 2011; 151: 1105-1108.

20 Yaghoobi P, Moghaddam MV, Nojeh A. Solar electron source and thermionic solar cell. AlP Adv 2012; 2: 042139

21 Arenal R, Löthman P, Picher M, Than T, Paillet M et al. Direct evidence of atomic structure conservation along ultra-long carbon nanotubes. J Phys Chem C 2012; 26: 14103-14107.

22 Steiner M, Freitag M, Tsang JC, Perebeinos V, Bol AA et al. How does the substrate affect the Raman and excited state spectra of a carbon nanotube? Appl Phys A 2009; 96: 271-282.

23 Li L, Guo W, Yan YZ, Lee S, Wang T. Label-free super-resolution imaging of adenoviruses by submerged microsphere optical nanoscopy. Light-Sci App/ 2013; 2: e104; doi:10.1038/Isa.2013.60.

24 Zhang X, Yang F, Zhao D, Cai L, Luan PS et al. Temperature dependent Raman spectra of isolated suspended single-walled carbon nanotubes. Nanoscale 2014; 6: 39493953.

25 Allen CS, Zhang C, Burnell G, Brown AP, Robertson J et al. A review of methods for the accurate determination of the chiral indices of carbon nanotubes from electron diffraction patterns. Carbon 2011; 49: 4961-4971.

26 Chu HB, Wang JY, Ding L, Yuan DN, Zhang Y et al. Decoration of gold nanoparticles on surface-grown single-walled carbon nanotubes for detection of every nanotube by surface-enhanced Raman spectroscopy. J Am Chem Soc 2009; 131: 1431014316 .
27 Zhang RF, Zhang YY, Zhang Q, Xie HH, Wang HD et al. Optical visualization of individual ultralong carbon nanotubes by chemical vapour deposition of titanium dioxide nanoparticles. Nat Commun 2013; 4: 1727.

28 Wang JT, Li TY, Xia BY, Jin X, Wei HM et al. Vapor-condensation-assisted optical microscopy for ultralong carbon nanotubes and other nanostructures. Nano Let 2014; 14: 3527-3533.

29 Pettes MT, Shi L. Thermal and structural characterizations of individual singledouble-, and multi-walled carbon nanotubes. Adv Funct Mater 2009; 19: 39183925.

30 Nugraha AR, Saito R, Sato K, Araujo PT, Jorio A et al. Dielectric constant model for environmental effects on the exciton energies of single wall carbon nanotubes. App Phys Lett 2010; 97: 091905.

31 Pop E, Mann D, Wang Q, Goodson KE, Dai HJ. Thermal conductance of an individual single-wall carbon nanotube above room temperature. Nano Lett 2006; 6: $96-100$.

$32 \mathrm{Hu}$ M, Shenogin S, Keblinski P, Raravikar N. Thermal energy exchange between carbon nanotube and air. Appl Phys Lett 2007; 90: 231905.

33 Carlborg CF, Shiomi J, Maruyama S. Thermal boundary resistance between singlewalled carbon nanotubes and surrounding matrices. Phys Rev B 2008; 78: 205406.

34 Qiu B, Wang Y, Zhao Q, Ruan XL. The effects of diameter and chirality on the therma transport in free-standing and supported carbon-nanotubes. Appl Phys Lett 2012; 100: 233105.

35 Cao AJ, Qu JM. Size dependent thermal conductivity of single-walled carbon nanotubes. J Appl Phys 2012; 112: 013503.

36 Hsu IK, Pettes MT, Aykol M, Chang CC, Hung WH et al. Direct observation of heat dissipation in individual suspended carbon nanotubes using a two-laser technique. J App/ Phys 2011; 110: 044328.

37 Talghader JJ, Gawarikar AS, Shea RP. Spectral selectivity in infrared thermal detection. Light-Sci App/ 2012; 1: e24; doi:10.1038/lsa.2012.24.

38 Cronin SB, Yin Y, Walsh A, Capaz RB, Stolyarov A et al. Temperature dependence of the optical transition energies of carbon nanotubes: the role of electron-phonon coupling and thermal expansion. Phys Rev Lett 2006; 96: 127403.

39 Nair RR, Blake P, Grigorenko AN, Novoselov KS, Booth TJ et al. Fine structure constant defines visual transparency of graphene. Science 2008; 320: 1308.

40 Xin H, Li B. Optical orientation and shifting of a single multiwalled carbon nanotube. Light-Sci App/2014; 3: e205; doi:10.1038/lsa.2014.86.

cc) (i) (-) $\odot$ This license allows readers to copy, distribute and transmit the cc) Contribution as long as it attributed back to the author. Readers may not alter, transform or build upon the Contribution, or use the article for commercial purposes. Please read the full license for further details at - http://creativecommons.org/licenses/ by-nc-nd/4.0/ 